\title{
Optimization of MKID Noise Performance Via Readout Technique for Astronomical Applications
}

\author{
Nicole G. Czakon ${ }^{a}$, James A. Schlaerth ${ }^{b}$, Peter K. Day ${ }^{c}$, Thomas P. Downes ${ }^{a}$, Ran P. Duan ${ }^{a}$, \\ Jiansong Gao ${ }^{d}$, Jason Glenn ${ }^{b}$, Sunil R. Golwala ${ }^{a}$, Matt I. Hollister ${ }^{a}$, Henry G. LeDuc ${ }^{c}$, \\ Benjamin A. Mazin ${ }^{e}$, Phillip R. Maloney ${ }^{b}$, Omid Noroozian ${ }^{a}$, Hien T. Nguyen ${ }^{c}$, Jack Sayers ${ }^{c}$, \\ Seth Siegel ${ }^{a}$, John E. Vaillancourt ${ }^{f}$, Anastasios Vayonakis ${ }^{a}$, Philip R. Wilson ${ }^{c}$, and Jonas \\ Zmuidzinas $^{a}$ \\ ${ }^{a}$ California Institute of Technology, Pasadena, CA 911125 USA; \\ ${ }^{b}$ Center for Astrophysics \& Space Astronomy, University of Colorado, Boulder, CO 80309 USA; \\ ${ }^{c}$ Jet Propulsion Laboratory, Pasadena, CA 91109 USA; \\ ${ }^{d}$ National Institute of Standards and Technology, Boulder, CO 80305 USA; \\ ${ }^{e}$ University of California, Santa Barbara, CA 93106 USA; \\ ${ }^{f}$ Stratospheric Observatory for Infrared Astronomy, Moffett Field, CA 94035 USA
}

\begin{abstract}
Detectors employing superconducting microwave kinetic inductance detectors (MKIDs) can be read out by measuring changes in either the resonator frequency or dissipation. We will discuss the pros and cons of both methods, in particular, the readout method strategies being explored for the Multiwavelength Sub/millimeter Inductance Camera (MUSIC) to be commissioned at the CSO in 2010. As predicted theoretically and observed experimentally, the frequency responsivity is larger than the dissipation responsivity, by a factor of $2-4$ under typical conditions. In the absence of any other noise contributions, it should be easier to overcome amplifier noise by simply using frequency readout. The resonators, however, exhibit excess frequency noise which has been ascribed to a surface distribution of two-level fluctuators sensitive to specific device geometries and fabrication techniques. Impressive dark noise performance has been achieved using modified resonator geometries employing interdigitated capacitors (IDCs). To date, our noise measurement and modeling efforts have assumed an onresonance readout, with the carrier power set well below the nonlinear regime. Several experimental indicators suggested to us that the optimal readout technique may in fact require a higher readout power, with the carrier tuned somewhat off resonance, and that a careful systematic study of the optimal readout conditions was needed. We will present the results of such a study, and discuss the optimum readout conditions as well as the performance that can be achieved relative to BLIP.
\end{abstract}

Keywords: Microwave Kinetic Inductance Detectors, Submillimeter, Nonlinearity,

\section{INTRODUCTION}

Microwave kinetic inductance detectors (MKIDs) are currently being used as the detecting elements of the Multiwavelength Sub/millimeter Inductance Camera (MUSIC). MKIDs are a promising new technology with a theoretical noise limit determined by generation-recombination noise. The white noise floor of our system is currently limited technologically by the noise temperature of our cryogenic HEMT and amplifier-gain fluctuations are introduced both by our HEMT as well as our warm electronics. It is desirable to read out the resonators with as high a power as possible in order to overcome the constant white noise contribution of the HEMT and drive down the resonator noise. As a detector resonance is driven at higher and higher readout powers, the total internal power increases,

$$
P_{\text {int }}=\frac{2}{\pi} \frac{Q^{2}}{Q_{c}} P_{\text {read }} \sim \frac{Q}{\pi} P_{\text {read }}
$$

Send correspondence to N.G.Czakon, E-mail: czakon@caltech.edu, Tel: 16263952648

Millimeter, Submillimeter, and Far-Infrared Detectors and Instrumentation for Astronomy V,
edited by Wayne S. Holland, Jonas Zmuidzinas, Proc. of SPIE Vol. 7741, 77410Q (c) 2010 SPIE · CCC code: $0277-786$ X/10/\$18 $\cdot$ doi: 10.1117/12.857866

Proc. of SPIE Vol. 7741 77410Q-1 


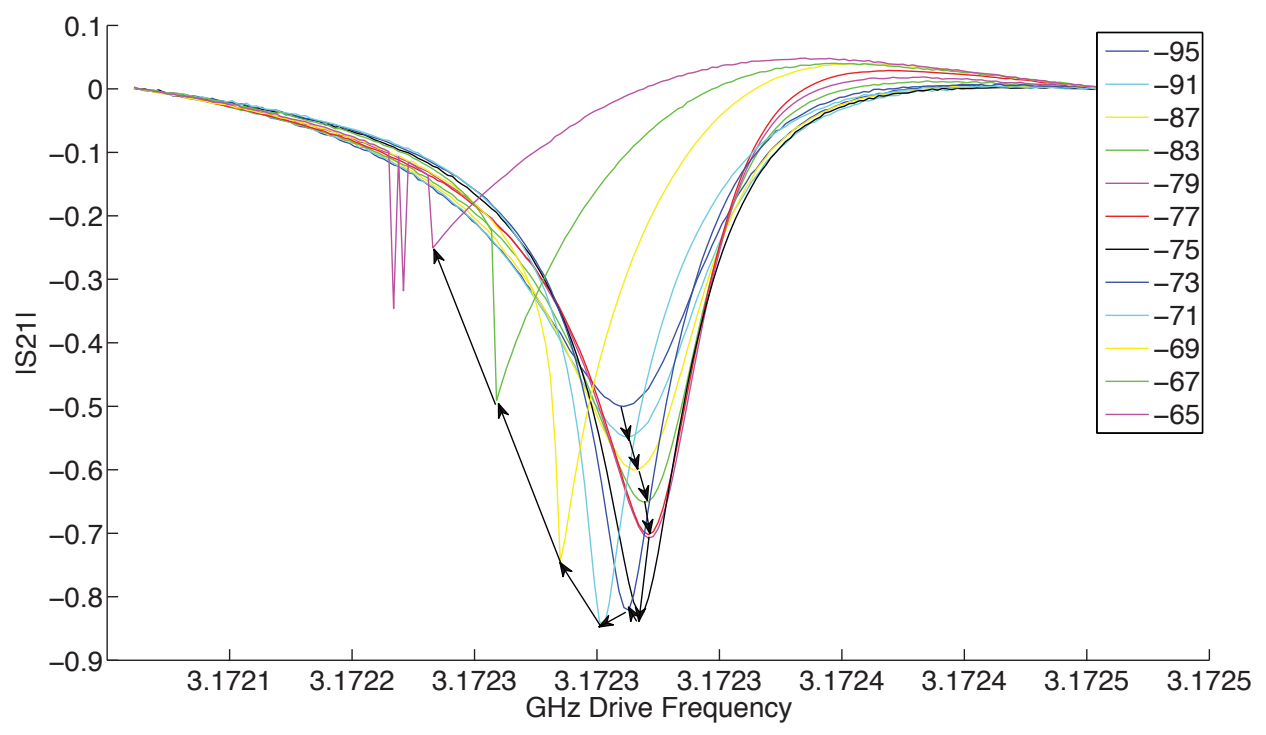

Figure 1: Change in S21 with Drive Power

and at a certain internal power level, the resonant frequency, quality factor and resonance shape become powerdependent. The amplitude of the transmitted signal is plotted in figure 1 for various readout powers. Ultimately the resonator becomes bistable and a discontinuity appears in the S21 curve. In addition to reducing the fractional contribution of the HEMT noise and resonator noise, signal responsivity can be increased by driving the resonators at higher readout powers. Such nonlinear amplification has been exploited in a variety of fields, such as with the Josephson bifurcation amplifier, and a detailed theoretical and experimental treatment of bifurcation gain has been established. ${ }^{1-3}$ With our current device, the noise contributions of both the HEMT amplifier and two-level systems on our substrate make it necessary to drive the resonators in a nonlinear regime to achieve background-limited photon (BLIP) detection. Optimal signal-to-noise performance is attained by tuning a resonator's readout power properly and exploiting the nonlinear responsivity of the device. Tholen et al. have done studies with superconducting $\mathrm{Nb}$ coplanar waveguide resonators, similar to the ones used in MuSIC, and they observed an amplification of $+22.4 \mathrm{~dB} .{ }^{4}$ We observe an increase in response of approximately $+13 \mathrm{~dB}$ but have decided to operate our detectors closer to the linear regime with only $+4 \mathrm{~dB}$ gain in responsivity.

\section{RESONANT LOOPS AND NOISE}

Some characteristics of the noise can be identified by plotting the data in the IQ-plane. As the readout frequency is stepped across a resonance, the output signal gains additional phase and sweeps out a resonance loop in the IQ plane such as in figure 2. Data is collected by tuning the readout frequency to resonance and monitoring the shift in phase and amplitude of the transmitted signal during the course of the observation. Specific properties of the individual noise contributions will affect the relative orientation of the noise to the resonance loop. The most familiar noise source is the Johnson-like white noise contribution from the HEMT. The power spectral density for this noise is

$$
S_{I Q}^{H E M T}(\nu)=k T_{n} Z_{0} / 2,
$$

where $\mathrm{k}$ is Boltzmann's constant and $Z_{0}$ is the impedance of the $50 \mathrm{Ohm}$ feedline. Uncorrelated HEMT white noise is a linear function of the amplifier noise temperature, which for the InP HEMTs that we have chosen to use is between $2-4 \mathrm{~K}$. The source of power-dependent amplifier-gain noise is different than the white noise, and due it partially being correlated, it can be removed fairly effectively from our signal. To obtain the highest mapping speeds, and to avoid systematics that often arise due to chopping, we choose our observation bandwidth to range from $1 \mathrm{mHz}$ to $1 \mathrm{~Hz}$ and excess amplifier-gain noise is still a concern since aggressive data cleaning methods 

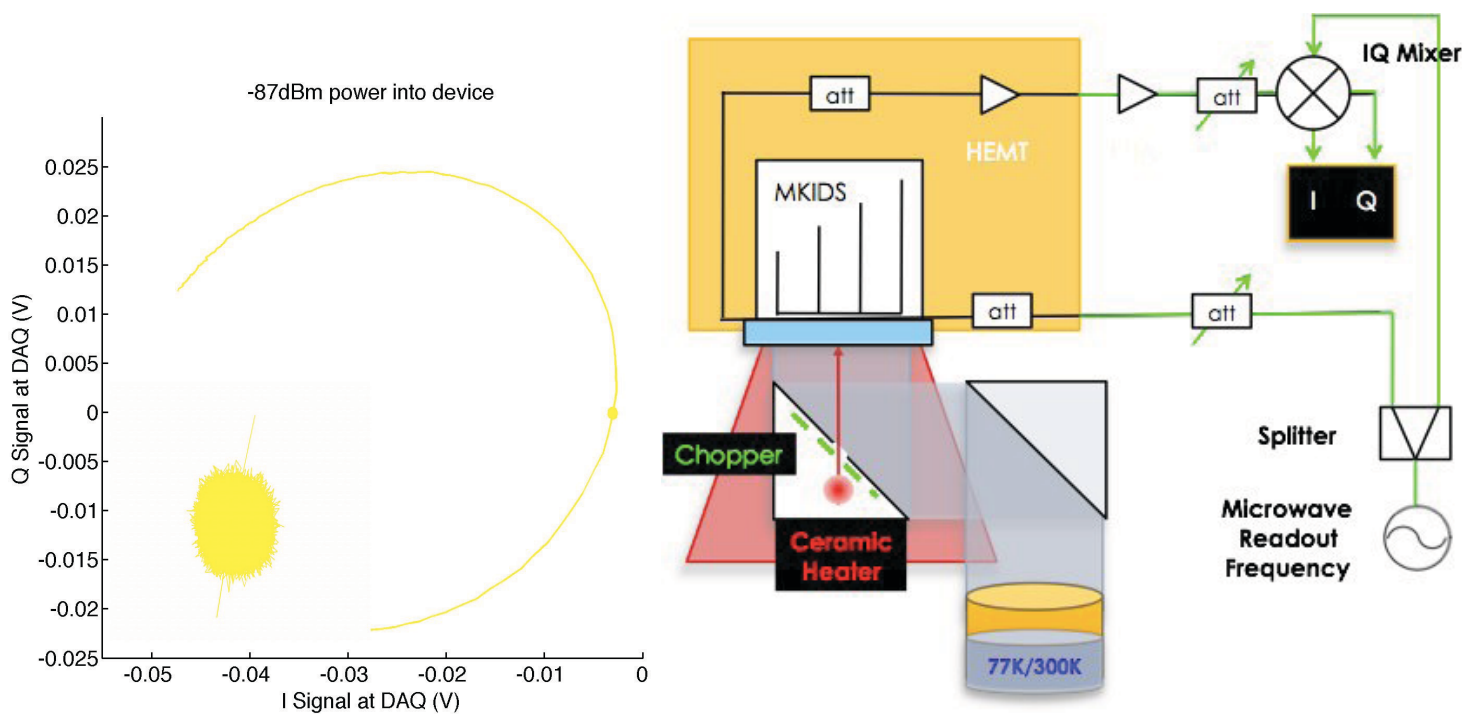

Figure 2: (Left) Sample Resonance Loop with an enlarged inset of the data noise ellipse. (Right) Configuration for Measuring the Predicted Response.

will affect the fidelity of the signal itself. For intermediate readout powers, device noise can still dominate our readout. Device noise manifests itself in the phase direction due to two-level system fluctuations at the surface of the dielectric which alter the effective capacitance of the resonant circuit. This can be seen in the inset of figure 2 where there is an elongation of the noise tangential to the IQ-loop. It should be noted that the resonance loop underestimates the scaling of the frequency to dissipation noise since it also includes $1 / f$ noise contributions from the amplifiers. which can be removed to a large extent with our software defined readout that was not employed for these sets of measurements. It has been established that TLS scale with noise according to $1 / \sqrt{P} .{ }^{5}$

The final major source of noise for MUSIC is sky noise. In our recent observation run at the Caltech Submillimeter Observatory (CSO) in May and June 2010, sky noise was often an order of magnitude higher than any type of detector or amplifier noise. Fortunately the MKID beams overlap fairly well in the Earth's atmosphere and the excess noise is removable by applying a weighted mean subtraction. ${ }^{6}$ Similar mean template subtraction has been successfully applied to sky data with MUSIC and sky noise has been reduced by about a factor of 20. Since the major source of sky noise is due to direct pickup by the antennas, this will change the quasiparticle density and result in noise along the signal direction. The signal direction can be determined by forming a vector from the frequency and the amplitude response. At low drive powers, the angle of the signal response with respect to the frequency and amplitude and direction is constant. However figure 3 shows that at higher drive powers, the relative size of the responses change, aligning more in the frequency direction.

To summarize, there are three major sources of noise that affect the performance of MUSIC: white HEMT noise, excess TLS noise in the frequency direction, and excess sky noise in the signal direction. Since resonator noise and white noise is not correlated across the detectors, it should be eliminated as much as possible. Optimal signal to noise can be achieved by choosing the projection axes properly.

\section{PREDICTING TELESCOPE NEP PERFORMANCE IN LAB}

The schematic of our lab setup to predict NEP performance is diagrammed in figure 2. Long timestreams of data are taken both with and without a chopped hot ceramic load placed in front of the dewar's window. The noise level is obtained from the unchopped power spectral densities (PSDs), while the response is obtained from the chopped PSDs within a narrow bandwidth of the chopper frequency. We calibrate the power emitted from the chopped source and subsequently calculate the predicted NEP using the formulism outlined below. To read out the array, we used a microwave synthesizer to generate a single frequency which is split into two parts. One part is sent through the dewar to read out the device while the other part is sent to the local oscillator port 


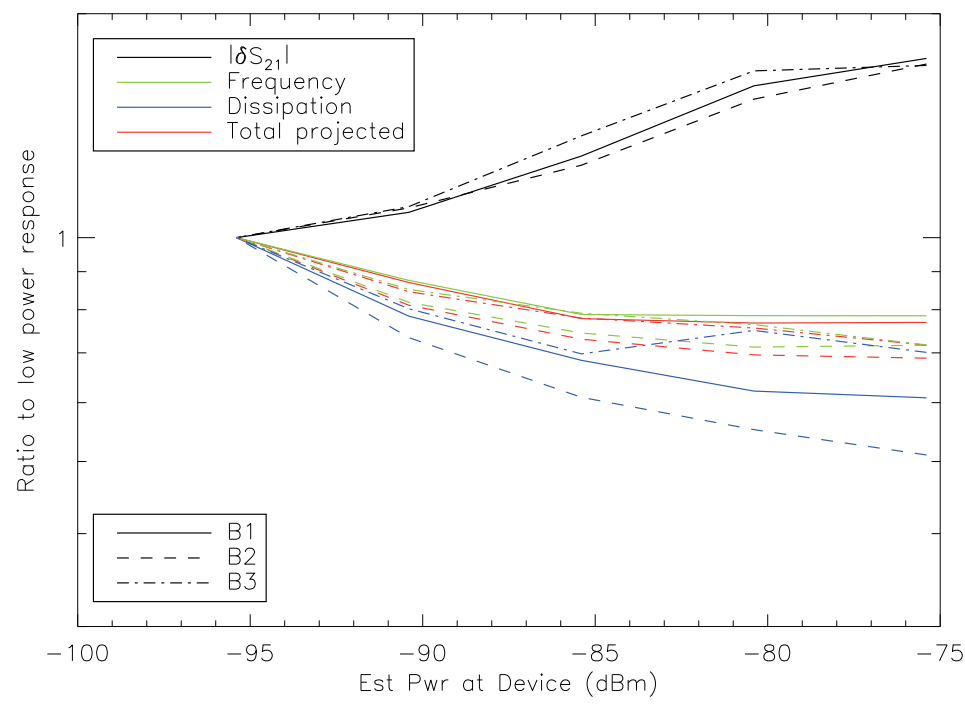

Figure 3: Change in relative frequency and dissipation response at higher drive powers for three different bands.

of an IQ mixer where it mixes the signal out of the dewar down to baseband. The I and Q outputs from the mixer are then sent to be recorded by a PCI-DAQ card. Balanced variable attenuators are located on the input and the output of the dewar in order to ensure that the signal fills the full dynamic range of the PCI-DAQ card for best performance. Our recently developed software-defined readout (SDR) was not yet available for these measurements so all measurements are taken one resonator at a time.

\subsection{Responsivity Measurements}

The hot load is very small compared to a single pixel's beam and the detectors only see a fractional difference in the background loading temperature while the source is chopped. Since the heater is not completely isothermal, an additional step is required to calibrate it's temperature. To this end, we take measurements using a beamfilling piece of Eccosorb ${ }^{\circledR}$ at both room and liquid nitrogen temperature. From the shift in resonant frequency for the two loads we obtain a measurement of the device's large signal responsivity. The theory for calibrating the chopped source to the hot/cold measurements can be found in James Schlaerth's thesis, ${ }^{7}$ in preparation, with an abbreviated version outline below. With the chopped source calibrated in the linear regime, the same source power is used to calculate the NEP for all drive powers.

Since the fractional change in quasiparticle density is a function of load, we must calibrate appropriately to find the responsivity for our chopped source which sees constant loading. To this end we note that the change in frequency for a resonator driven in the linear regime is proportional to the change in quasiparticle density.

$$
\begin{aligned}
n_{q p}=\sqrt{\frac{\eta k_{B} T_{1}(\Delta \nu)}{R V \Delta}} \\
\frac{\delta f}{\delta T}=\frac{d f}{d n_{q p}}\left(\sqrt{\frac{\eta k_{B} T_{1}(\Delta \nu)}{R V \Delta}}-\sqrt{\frac{\eta k_{B} T_{2}(\Delta \nu)}{R V \Delta}}\right) \frac{1}{T_{1}-T_{2}} \\
=\operatorname{const.} \frac{1}{\sqrt{T_{1}}+\sqrt{T_{2}}} .
\end{aligned}
$$

Where $n_{q p}$ is the quasiparticle density, $T_{1}$ and $T_{2}$ are the temperatures of the two different loads, $\eta$, is the efficiency at which quasiparticles are generated, $\Delta \nu$ is the effective bandwidth of the MKID, $R$ is the quasiparticle recombination rate, and $\Delta$ is the gap energy required to break a Cooper pair. In equation 5 we see that response 
has a relatively simple temperature dependence. The warmer either load, the smaller the response. When we compare the predicted response of hot/cold response measurements to what we expect at the sky or from the chopped source, we can simply take a ratio of the responsivities to obtain the constant,

$$
\begin{aligned}
\frac{\delta f_{s k y}}{\delta T_{s k y}} & =\frac{\delta f_{H C}}{\delta T_{H C}} \frac{\sqrt{T_{H}}+\sqrt{T_{C}}}{\sqrt{T_{s k y}}+\sqrt{T_{s k y}+\epsilon}} \\
& \approx 1.5 \frac{\delta f_{H C}}{\delta T_{H C}}
\end{aligned}
$$

In the last part of the equation 7 we have made the assumption that the hot and cold temperature loads are $300 \mathrm{~K}$ and $77 \mathrm{~K}$ respectively, and $77 \mathrm{~K}$ for the small signal response, the additional loading is a small perturbation which can be ignored.

Since the hot cold loads can be approximated as blackbodies in the Rayleigh-Jeans limit, $P=2 k_{b} T \Delta \nu$, they serve as the calibrators. The bandwidths of the $1.3 \mathrm{~mm}, 1.1 \mathrm{~mm}$, and $.85 \mathrm{~mm}$ bands are $55 \mathrm{GHz}, 45 \mathrm{GHz}$, and 30 $\mathrm{GHz}$, respectively. Converting temperature in the denominator to chopped load power,

$$
\frac{\delta f_{s k y}}{\delta P}=\frac{\delta f_{H C}}{\delta T_{H C}} \frac{\sqrt{T_{H}}+\sqrt{T_{C}}}{2 \sqrt{T_{s k y}}} \frac{1}{2 k_{B} \Delta \nu},
$$

and from this, a calculation of the chopped signal power,

$$
\Delta P \approx \Delta f_{s k y} \frac{1}{\frac{\delta f_{H C}}{\delta T_{H C}}} \frac{4 \sqrt{T_{s k y}} k_{B} \Delta \nu}{\sqrt{T_{H}}+\sqrt{T_{C}}} .
$$

Another important difference between a small signal load and a sky load is the presence of dark response. In summer of 2009 our group noticed that our detectors have a significant amount of dark response. We attributed this to direct pickup with our interdigitated capacitors (IDCs) that had been recently incorporated with the design to reduce the magnitude of the TLS noise. To reduce the dark coupling we simulated and included stepped-impedance filters between the IDCs and the aluminum section, and this got rid of some of the excess dark loading, but not all of it. Some of the residual dark response was due to tile heating caused by poor heat sinking - a consequence of the larger array designs. ${ }^{8}$ Tile heating was reduced by switching from aluminum to gold bonds and using a fused quartz antireflection plate instead of a crystalline quartz one. An equally effective solution had been to borrow and install a $14 \mathrm{icm}$ dielectric filter to block out excess IR pickup. The additional gold bonds and quartz AR tile were almost as effective as the dielectric filter in eliminating tile heating. Tile temperatures can be approximated by fitting data to the Mattis-Bardeen theory which suggest negligent amounts of tile heating. Even with all of these additional measures, the dark response was still $1 / 3$ or $1 / 2$ the response of the lights for band 1 and band 3 respectively. There is still unwanted residual direct pickup and we are currently investigating the sources. Even in the absence of response, excess load is undesirable since it can decrease the resonator responsivity by as much as a factor of 2 between $300 \mathrm{~K}$ and $77 \mathrm{~K}$ load.

The mean values of lab hot/cold responsivities are: .352,.330,.268, and $.151 \mathrm{kHz} / \mathrm{K}$ for the $1.3 \mathrm{~mm}, 1.1 \mathrm{~mm}$, $.85 \mathrm{~mm}$, and dark bands. Since we measured the dark response to be negligible in the far field from our sky dip analysis, we can simply subtract off the dark response. Mean responsivities with the dark subtracted are: $.201, .179$, and $.117 \mathrm{kHz} / \mathrm{K}$ for $1.3 \mathrm{~mm}, 1.1 \mathrm{~mm}, .85 \mathrm{~mm}$ bands respectively. The responsivities are a function of loading which can change by tens of Kelvins depending on the amount of precipitable water vapor in the atmosphere. By combining all these responsivities together for lab measurements we get the following fractional frequency shifts per Watt in sky and in the lab as: .618, .401, and .664 kHz/pW sky, and .306, 1.99, and $.329 \mathrm{kHz} / \mathrm{pW}$ in the lab. The increase in responsivity is due the increased emissivity of a blackbody at higher frequencies for the loading temperatures that we're interested in. Converting this to Jansky's gives 5.13, 4.56, and $2.99 \mathrm{~Hz}$ per Jansky, or, .1709, .1521, and .09961e-8 ( $\Delta \mathrm{f} / \mathrm{f}) / \mathrm{Jy}$. 

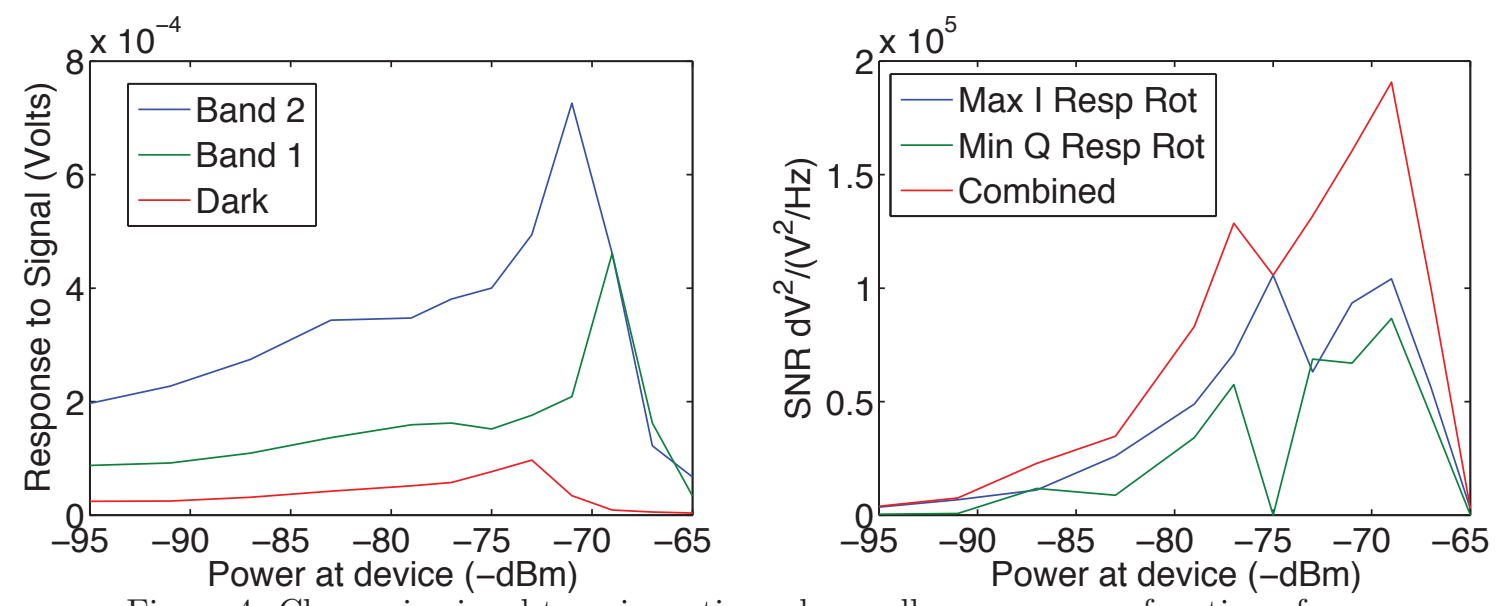

Figure 4: Change in signal-to-noise ratio and overall response as a function of power

\section{CHANGING RESPONSE AND S/N USING DRIVE POWER}

We too have made measurements of the signal power and signal to noise as a function of drive power and the results are presented in figure 4 . The response looks similar to one of the device in the Tholen paper. ${ }^{4}$ Recall that since the attenuation is balanced on the input and output of the dewar, any increase in response is solely due to the changing properties of the resonator in the nonlinear regime. In both figures, I simply plot the phase-noise which has much higher signal response than the amplitude direction. During the Spring 2010 observing run, we drove our resonators at $-75 \mathrm{dBm}$ estimated power at the device, which is a regime where signal to noise is high, but before we start to see too many nonlinear effects.

Readout power is not the only method in which one can optimize resonator signal to noise. Magnetic fields can be used to tune the frequency and quality factor microresonators which would correspond to change in responsivity and we have not yet explored this degree of freedom. ${ }^{9}$

\subsection{Calculating NEP}

There are several assumption that go into finding an NEP number for the given conditions. First, NEPs calculated for the telescope are different than those calculated for lab. This is due to different loading conditions and different levels of dark response. We have calculated that the excess load on the resonators using the chopper setup in lab is approximately $35 \mathrm{~K}$, making the entire load on the resonators $100 \mathrm{~K}$. To measure the amount of optical power that reaches the detectors, we assume the Rayleigh-Jeans limit of the blackbody spectrum and multiply the entire equation by our expected window-to-device optical efficiency.

$$
\begin{aligned}
Q_{0} & =2 \epsilon k_{b} T_{\text {load }} \Delta \nu \\
\mathrm{NEP} & =\sqrt{2 Q_{0} h \nu_{0}+Q_{0}^{2} / \delta \nu} \\
N E P_{\text {window }} & =\sqrt{2 Q_{0} h \nu_{0}+Q_{0}^{2} / \delta \nu} / \epsilon
\end{aligned}
$$

There is some variability in the efficiency of our detectors, but we have measured the response to be between $1.5 \%$ and $3 \%$. Again, dark loading adds an additional element of confusion. Although the optical efficiency is small for a point source, the dark pickup will increase the overall amount of power reaching the detector, and therefore the optical efficiency. Therefore, to calculate BLIP we must use the overall optical efficiency including the dark response, which is about $5 \%$. For our device, we calculate BLIP at the device to be approximately, $5 \cdot 10^{-17}$, for both bands, which is close to the minimum of our NEP measurements in figure 5. 


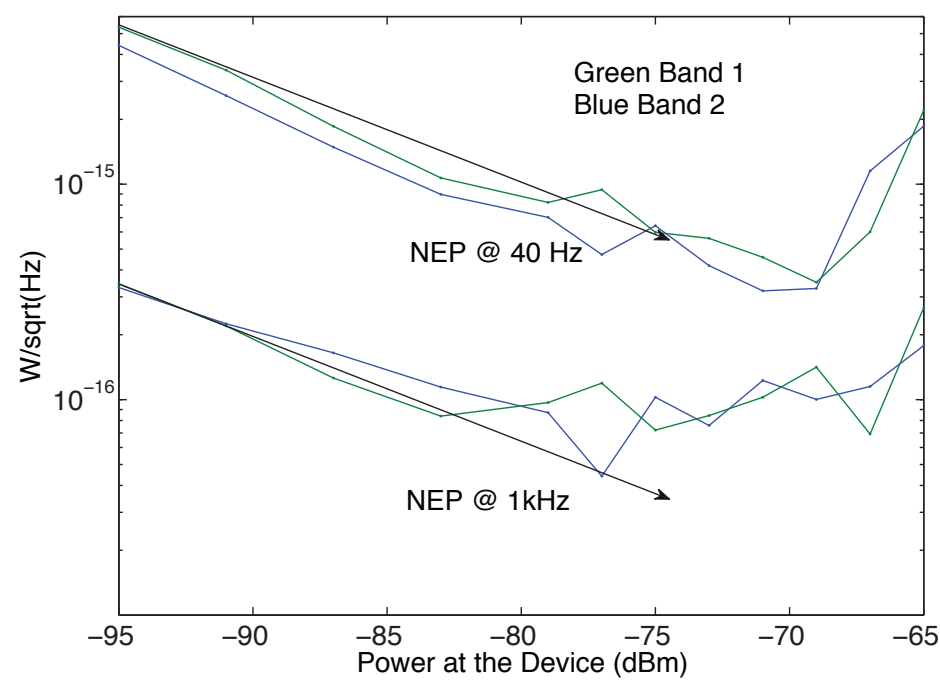

Figure 5: NEPs calculated for various powers

\subsection{Characterization of the Noise}

A quick glance at figure 5 would suggest that the increase in efficiency is simply due to the decrease in HEMT noise. However, this is not the case. For the very lowest powers that are measured, $-95 \mathrm{dBm}$ at the device, both frequency and dissipation response are dominated by HEMT noise. Resonator noise starts to dominate at the $-91 \mathrm{dBm}$ power level and starts to fall off with the $1 / \sqrt{P}$. The decrease in NEP is mostly due to the increase in responsivity of the resonators.

\section{CONCLUSIONS}

By driving the resonators of the existing MUSIC prototype camera in a slightly nonlinear regime, we are able to increase the signal size by a factor of 3 and reduce the NEP by a factor of 10 . This regime ensures both low HEMT noise and low resonator noise contributions. The collected data suggests that at high frequencies we are very close to background limited performance.

\section{ACKNOWLEDGMENTS}

The MuSIC project is supported by NSF grant AST-0705157 to the University of Colorado, NASA grants NNGC06C71G and NNX10AC83G to Caltech, the Gordon and Betty Moore Foundation, and the JPL Research and Technology Development Fund. We are grateful to the Xilinx corporation for their generous donation of the FPGAs needed for the readout electronics. J. Sayers was supported by a NASA Postdoctoral Program Fellowship and J. Schlaerth and N. Czakon were supported by the NASA Graduate Student Researchers Program Fellowships. 


\section{REFERENCES}

[1] Wiesenfeld, K. and McNamara, B., "Period-doubling systems as small-signal amplifiers," Physical Review Letters 55, 13-16 (July 1985).

[2] Wiesenfeld, K. and McNamara, B., "Small-signal amplification in bifurcating dynamical systems," Phys. Rev. A 33, 629-642 (1986).

[3] Vijay, R., Devoret, M., and Siddiqi, I., "Invited review article: The josephson bifurcation amplifier," Rev. Sci. Instr. 80, 111101-1:17 (2009).

[4] Tholen, E. A., Ergul, A., Doherty, E. M., Weber, F. M., Gregis, F., and Haviland, D. B., "Nonlinearities and parametric amplification in superconducting coplanar resonators," Appl. Phys. Letts. 90, 253509-1:3 (2007).

[5] Gao, J., Mazin, B., Daal, M., Day, P., LeDuc, H., and Zmuidzinas, J., "Power dependence of phase noise in microwave kinetic inductance detectors," in [Astronomical Telescopes and Instrumentation], Zmuidzinas, J., Holland, W. S., Withington, S., and Duncan, W. D., eds., Proc. SPIE, 627509 (2006).

[6] Sayers, J., Golwala, S., Ade, P., Aguirre, J., Bock, J., Edgington, S., Glenn, J., Goldin, A., Haig, D., Lange, A., Laurent, G., Mauskopf, P., Nguyen, H., Rossinot, P., and Schlaerth, J., "Studies of millimeter-wave atmospheric noise above mauna kea," Astrophysical Journal 708, 1674-1691 (jan 2010).

[7] Schlaerth, J., [Microwave Kinetic Inductance Detector Camera Development for Millimeter-Wave Astrophysics, in preparation], Boulder, Colorado, University of Colorado (2010).

[8] Schlaerth, J., Czakon, N., Day, P., Downes, T., Duan, R., Gao, J., Glenn, J., Golwala, S., Hollister, M., LeDuc, H., Mazin, B., Maloney, P., Noroozian, O., Nguyen, H., Sayers, J., Siegel, S., Vaillancourt, J., Vayonakis, A., Wilson, P., and Zmuidzinas, J., "Mkid multicolor array status and results from democam," in [Astronomical Telescopes and Instrumentation], Zmuidzinas, J. and Holland, W., eds., Proc. SPIE, 7741-8 (2010).

[9] Healey, J., Lindstrom, T., Colclough, M., Muirhead, C., and Tzalenchuk, A. Y., "Magnetic field tuning of coplanar waveguide resonators," Appl. Phys. Letts. 93, 043513-1:3 (2008).

[10] Duan, R., Czakon, N., Day, P., Downes, T., Duan, R., Gao, J., Glenn, J., Golwala, S., Hollister, M., LeDuc, H., Mazin, B., Maloney, P., Noroozian, O., Nguyen, H., Sayers, J., Schlaerth, J., Siegel, S., Vaillancourt, J., Vayonakis, A., Wilson, P., and Zmuidzinas, J., "An open-source software-defined radio readout for mkids," in [Astronomical Telescopes and Instrumentation], Zmuidzinas, J. and Holland, W., eds., Proc. SPIE, 7741-67 (2010). 